\title{
Neck circumference as an independent indicator of visceral obesity in a Chinese population
}

\author{
Li Zhao $^{1 \dagger}$, Guolan Huang ${ }^{2+}$, Fangzhen Xia', Qin Li', Bing Han', Yi Chen', Chi Chen', Dongping Lin', \\ Ningjian Wang ${ }^{1 *}$ and Yingli $\mathrm{Lu}^{1,2^{*}}$
}

\begin{abstract}
Background: Neck circumference (NC) was reported to be associated with visceral obesity in some specific subjects. However, no studies have reported whether NC could identify visceral obesity in the general population. Here, we mainly aimed to explore whether NC is suitable to identify visceral obesity in the general population.

Methods: Our data were from a cross-sectional survey on the prevalence of metabolic diseases and risk factors in East China from 2014 to 2015. A total of 9366 participants aged 18-93 were identified for analysis. Anthropometric indices, biochemical parameters and clinical characteristics were measured. The NC values were quartered according to sex. Spearman's correlation coefficient was employed to test the correlations between different variables. Linear regression and logistic regression were conducted to explore the relationship of NC with visceral adiposity indices and visceral obesity.

Results: Among the 9366 participants, 3938 (42.05\%) were male and 5428 (57.95\%) were female. NC had a positive correlation with the visceral adiposity indices, regardless of sex. In all quartiles of NC, in both men and women, as $N C$ values increased, the values of all the fatness indices showed a tendency to increase (all $P<0.001$ ). After full adjustment for demographic variables and metabolic factors, linear regression showed that NC was still associated with the fatness indices for visceral obesity (all $P<0.001$ ). In addition, logistic analysis showed that a larger NC was associated with a higher risk of visceral obesity in both males (OR 32.34, 95\% Cl 24.02-43.53; $P<0.001)$ and females (OR 21.43, 95\% Cl 17.30-26.55; $P<0.001$ ) after adjusting for potential confounding factors.
\end{abstract}

Conclusion: NC can be a supplemental indicator for identifying visceral obesity in the general Chinese population.

Keywords: Neck circumference, Visceral obesity, Lipid metabolism

\section{Background}

According to the epidemiological data from the World Health Organization (WHO), there are more than half a billion adults with overweight or obesity [1]. Obesity, especially visceral obesity, is a well-known risk factor for many disorders, such as metabolic disease [2], hemodynamic, endothelial, inflammatory [3] and even physiological disorders. Thus, continuous monitoring and early detection of obesity can prevent the onset

\footnotetext{
* Correspondence: wnj486@126.com; luyingli2008@126.com

${ }^{\dagger}$ Equal contributors

${ }^{1}$ Institute and Department of Endocrinology and Metabolism, Shanghai Ninth People's Hospital, Shanghai JiaoTong University School of Medicine, Shanghai 200011, China

Full list of author information is available at the end of the article
}

of its adverse outcomes. However, the distribution of adipose tissue plays a more important role than the amount in the development of these disorders [4].

Currently, the sophisticated methods to assess visceral adiposity include dual-energy X-ray absorptiometry (DEXA), computed tomography (CT) and magnetic resonance imaging (MRI) [5]. However, DEXA, CT and MRI are impractical for screening the general population, since they require expensive and specialized equipment, and patients are exposed to radiation. In this regard, many indices to estimate central or visceral obesity have been suggested. Among them, classical parameters such as waist circumference (WC), waist-to-hip ratio (WHR) and waist-to-height ratio (WHtR) are the most popular indices

(C) The Author(s). 2018 Open Access This article is distributed under the terms of the Creative Commons Attribution 4.0 International License (http://creativecommons.org/licenses/by/4.0/), which permits unrestricted use, distribution, and 
that are widely applied in clinical settings. More recent indices, including the visceral adiposity index (VAI), the lipid accumulation product (LAP), the abdominal volume index (AVI) and the conicity index (Cindex), which are calculated on the basis of simple data such as triglycerides (TG), high-density lipoprotein (HDL), body mass index (BMI), weight, height, WC, and hip circumference (HC), have also been introduced $[6,7]$. In particular, the VAI has proven to be an indicator of adipose distribution and function in more than 30 publications [8]. However, BMI mainly reflects overall obesity, and WC varies with the phases of respiration and fullness of the stomach. Moreover, both WHR and WC are based on longitudinal measures that are not very reliable for estimating central obesity in subjects who develop pendulum abdomen, in which the line of the umbilicus falls below the line of the hip. Given this information, all of these indices have certain limitations.

Deposition of fat around the neck is a unique phenomenon that depicts upper body subcutaneous adipose tissue. Measurement of the neck circumference (NC) has been identified as a surrogate marker for determining upper-body subcutaneous fat distribution. It is low-cost, reliable, noninvasive, reproducible and unaffected by phases of respiration or stomach fullness. $\mathrm{NC}$ has been proven to be closely associated with other anthropometric parameters (e.g., BMI and WC) and various metabolic risk factors [9-12]. Previous studies also showed that $\mathrm{NC}$ or neck fat content is positively correlated with visceral fat content; however, these studies were conducted in HIV-infected individuals, with small sample sizes or in combination with other diseases, limiting the degree to which the findings can be generalized [11, 13, 14]. Here, we aimed to find whether $\mathrm{NC}$ is a good indicator for evaluating visceral obesity in the general population by comparing the relationship between $\mathrm{NC}$ and the fatness indices expressing visceral fat distribution.

\section{Methods}

\section{Study population}

SPECT-China is a cross-sectional survey on the prevalence of metabolic diseases and risk factors in East China (ChiCTRECS-14,005,052, www.chictr.org. $\mathrm{cn})$. The details of the study design have been described previously [15-17]. In brief, the study was performed from February 2014 to December 2015. Twenty-two sites in Shanghai, Jiangxi Province, Zhejiang Province, Jiangsu Province and Anhui Province were selected using a stratified cluster sampling method. Adults aged 18 years and older who were Chinese citizens and had lived at their current residence for 6 months or longer were invited to participate in our study. Those with severe communication problems, with acute illness or who were unwilling to participate were excluded from the study. A total of 10,798 residents participated in this investigation. After exclusion of participants who were completely missing laboratory results $(n=191)$, were missing questionnaire data $(n=159)$, or were younger than 18 years old $(n=7), 10,441$ subjects were enrolled in the SPECT-China study. Candidates with missing NC or WC measurements $(n=459)$ and some biochemical indices (e.g., insulin or TG levels, $n=$ 352) were also excluded. In addition, patients having neck diseases including goiter or other neck masses or deformities identified with ultrasound diagnosis ( $n$ $=232$ ) or with long-term use of hormone replacement therapy $(n=32)$ were excluded. Finally, a total number of 9366 participants aged 18-93 years were analyzed in this study (Fig. 1).

The study design protocol was approved by the Ethics Committee of Shanghai Ninth People's Hospital, Shanghai JiaoTong University School of Medicine. All procedures were in accordance with the ethical standards of the responsible committee on human experimentation (institutional and national) and with the Helsinki Declaration of 1975 , as revised in 2008. Written informed consents were obtained from all patients.

\section{Clinical and anthropometric evaluation}

At every site, the same trained staff collected all the data according to a standard protocol. Trained interviewers used a questionnaire to collect information on demographic characteristics, medical history and lifestyle risk factors [18]. Current smoking was defined as having smoked at least 100 cigarettes in one's lifetime and currently smoking cigarettes, and current drinking was defined as alcohol intake more than once per month during the past 12 months [18]. Body weight, height, WC, HC and blood pressure were measured with the use of standard methods as described previously [12, 18]. NC was measured below the cricoid cartilage, and afterwards, at the level of the mid-cervical spine [19]. BMI was calculated as the weight in kilograms divided by the height in meters squared. WHR and WHtR were calculated as waist circumference divided by hip circumference and height, respectively. The VAI, LAP, AVI and Cindex were calculated as follows $[6,7]$ :

$$
\begin{aligned}
\text { Males : VAI } & =\mathrm{WC}(\mathrm{cm}) /\left[39.68+1.88 \times \mathrm{BMI}\left(\mathrm{kg} / \mathrm{m}^{2}\right)\right] \\
& \times[\mathrm{TG}(\mathrm{mmol} / \mathrm{L}) / 1.03] \times[1.31 / \mathrm{HDL}(\mathrm{mmol} / \mathrm{L})] \\
\mathrm{LAP} & =[\mathrm{WC}(\mathrm{cm})-65] \times \mathrm{TG}(\mathrm{mmol} / \mathrm{L})
\end{aligned}
$$




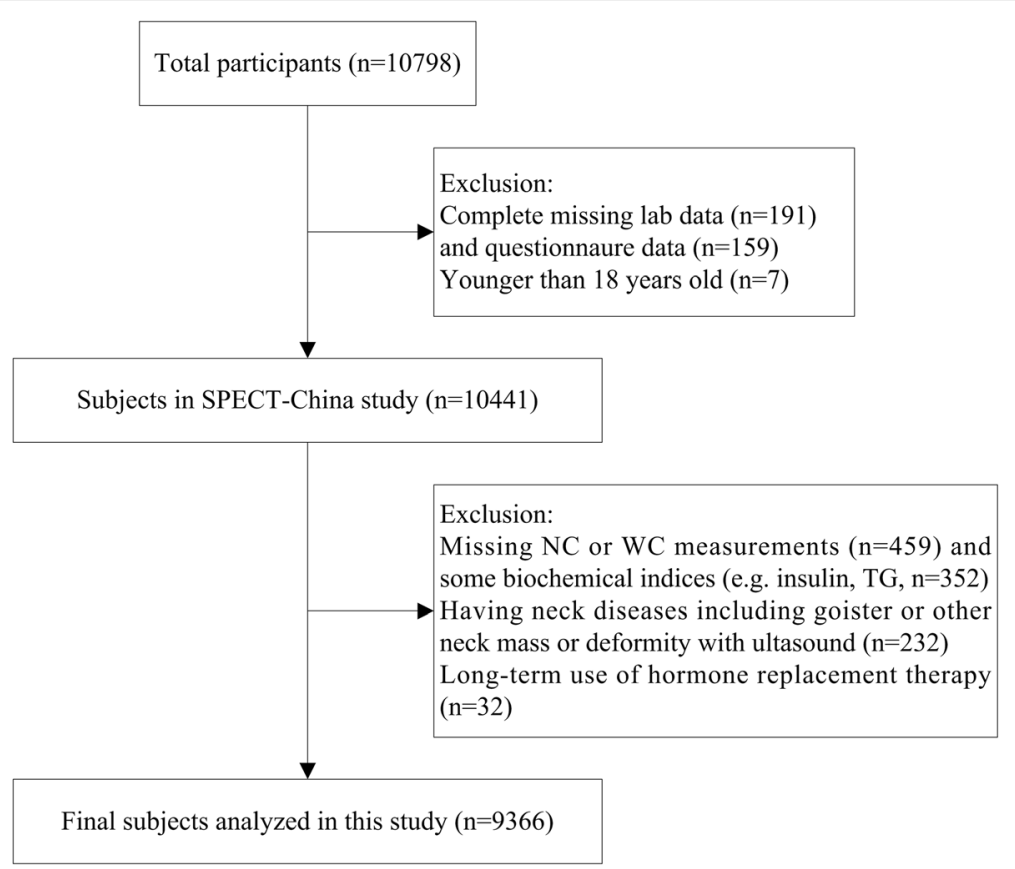

Fig. 1 Flowchart of participants selected from SPECT-China

$$
\text { Females : VAI } \begin{aligned}
& =\mathrm{WC}(\mathrm{cm}) /\left[36.58+1.89 \times \mathrm{BMI}\left(\mathrm{kg} / \mathrm{m}^{2}\right)\right] \\
& \times[\mathrm{TG}(\mathrm{mmol} / \mathrm{L}) / 0.81] \times[1.52 / \mathrm{HDL}(\mathrm{mmol} / \mathrm{L})] \\
\mathrm{LAP} & =[\mathrm{WC}(\mathrm{cm})-58] \times \mathrm{TG}(\mathrm{mmol} / \mathrm{L}) .
\end{aligned}
$$

For both males and females : AVI

$$
\begin{aligned}
= & {\left[2 \mathrm{~cm} \times \mathrm{WC}^{2}(\mathrm{~cm})^{2}+0.7 \mathrm{~cm} \times(\mathrm{WC}-\mathrm{HC})^{2}\right] } \\
& / 1000 \text { Cindex }=\mathrm{WC}(\mathrm{m}) \\
& / 0.109 \times \sqrt{ }[\text { weight }(\mathrm{kg}) / \operatorname{height}(\mathrm{m})] .
\end{aligned}
$$

\section{Biochemical measurements}

Peripheral venous blood samples were drawn after an overnight fast of at least $8 \mathrm{~h}$. The blood samples for the plasma glucose test were collected into vacuum tubes with the anticoagulant sodium fluoride and centrifuged within $1 \mathrm{~h}$ after collection. All blood samples were stored at $-20{ }^{\circ} \mathrm{C}$ after collection and then shipped by air within 2-4 h on dry ice to a central laboratory, which was certified by the College of American Pathologists. Glycated hemoglobin (HbA1c) was assessed by high-performance liquid chromatography (MQ-2000PT, China). Fasting plasma glucose (FPG), alanine aminotransferase (ALT) and a lipid profile including total cholesterol (TC), TG, low-density lipoprotein (LDL) and HDL were measured by a BECKMAN COULTER AU 680 (Germany). Insulin was detected by the chemiluminescence method (Abbott i2000 SR, USA). Then, insulin resistance was estimated by the homeostatic model assessment (HOMA-IR) index: [FINS $(\mathrm{mIU} / \mathrm{L}) \times$ FPG $(\mathrm{mmol} / \mathrm{L})] / 22.5$.

\section{Definition of variables}

Visceral obesity was defined as a waist circumference $\geq$ $90 \mathrm{~cm}$ in males and $\geq 80 \mathrm{~cm}$ in females [18].

\section{Statistical analysis}

Data analyses were performed with the software package SPSS Statistics, Version 22 (IBM Corporation, Armonk, NY, USA). Normally distributed data were expressed as the means $\pm \mathrm{SD}$, whereas continuous variables with a skewed distribution were summarized as the median with interquartile range $(25 \%, 75 \%)$ and were logarithmically transformed before analysis. To compare the differences between groups, one-way analysis of variance (ANOVA) was used for continuous variables with a Gaussian distribution, and the Kruskal-Wallis test was used for variables with a skewed distribution. Spearman's correlation coefficient was employed to test the correlations between different variables. The association of $\mathrm{NC}$ (independent variable) with fatness indices including WC, WHR, WHtR, VAI, LAP, AVI and Cindex (dependent variables) was assessed by linear regression. Model 1 did not adjust for any factors. Model 2 adjusted for age, smoking, drinking, HbA1c, TC, LDL and SBP. Binary logistic regression analysis was also conducted to explore the association of NC with visceral obesity. Similarly, Model 1 did not adjust for any factors. Model 2 
adjusted for age, smoking, drinking, HbA1c, TC, LDL and SBP. A two-sided $P$ value $<0.05$ was considered statistically significant.

\section{Results}

\section{Characteristics of study participants}

This study recruited 9366 subjects whose mean age was $53.04 \pm 13.02$ years. Among them, 3938 (42.05\%) were male and 5428 (57.95\%) were female, with a slight female preponderance ( $\mathrm{F}: \mathrm{M}=1.4: 1)$. The characteristics of the study participants according to the quartile of $\mathrm{NC}$ were summarized in Tables 1 and 2 . The mean value of $\mathrm{NC}$ was $33.52 \pm 3.29 \mathrm{~cm}$ in the total participants, and men had a mean NC $4.0 \mathrm{~cm}$ wider than women $(35.84 \pm$ 2.75 vs $31.84 \pm 2.54 \mathrm{~cm}$ ). The quartile ranges of $\mathrm{NC}$ in males were $\leq 34,34-36,36-38$ and $>38 \mathrm{~cm}$. Males with a larger NC had higher values for weight, WC, HC, BMI, WHR, WHtR and blood pressure and higher levels of FPG, HbA1c, FINS, HOMA-IR, TG, TC, LDL and ALT (all $P<0.001$ ). The indices (VAI, LAP, AVI and Cindex) assessing visceral fat accumulation also showed a graded increase as NC increased (all $P<0.001$ ). At the same time, the quartile ranges for $\mathrm{NC}$ in females were $\leq$ $30,30-32,32-33$ and $>33 \mathrm{~cm}$. Females with a larger NC also had higher values for metabolic parameters and the fatness indices (all $P<0.001$ ). In contrast, the participants with a larger NC displayed lower levels of $\mathrm{HDL}$, both in males and females (all $P<0.001$ ).

\section{Correlation coefficients for NC}

Correlation analysis demonstrated that $\mathrm{NC}$ had a positive correlation with all the fatness indices for both overall obesity and visceral obesity, including BMI, WC, WHR, WHtR, VAI, LAP, AVI and Cindex, regardless of sex (all $P<0.001$ ). In addition, NC was positively correlated with blood pressure, FPG, HbA1c, FINS, HOMA-

Table 1 Characteristics of study participants according to neck circumference quartiles in males

\begin{tabular}{|c|c|c|c|c|c|}
\hline \multirow[t]{2}{*}{ Male } & \multicolumn{4}{|c|}{ Neck circumference $(\mathrm{cm})$} & \multirow[t]{2}{*}{$P$} \\
\hline & $\leq 34$ & $34-36$ & $36-38$ & $>38$ & \\
\hline$N$ & 1240 & 1152 & 903 & 643 & \\
\hline Age (y) & $55.55 \pm 13.78$ & $52.95 \pm 13.43$ & $53.00 \pm 12.23$ & $52.83 \pm 12.05$ & $<0.001$ \\
\hline Weight (kg) & $61.64 \pm 8.22$ & $68.52 \pm 7.75$ & $74.69 \pm 7.87$ & $81.77 \pm 9.71$ & $<0.001$ \\
\hline WC $(\mathrm{cm})$ & $77.75 \pm 7.91$ & $82.87 \pm 7.38$ & $88.12 \pm 7.86$ & $93.75 \pm 7.79$ & $<0.001$ \\
\hline $\mathrm{HC}(\mathrm{cm})$ & $89.87 \pm 5.47$ & $93.66 \pm 5.37$ & $96.91 \pm 4.96$ & $100.44 \pm 6.23$ & $<0.001$ \\
\hline BMI $\left(\mathrm{kg} / \mathrm{m}^{2}\right)$ & $22.38 \pm 2.60$ & $24.31 \pm 2.40$ & $26.20 \pm 2.40$ & $28.49 \pm 2.86$ & $<0.001$ \\
\hline WHR & $0.87 \pm 0.07$ & $0.89 \pm 0.07$ & $0.91 \pm 0.07$ & $0.93 \pm 0.07$ & $<0.001$ \\
\hline $\mathrm{WHtR}$ & $0.47 \pm 0.05$ & $0.49 \pm 0.05$ & $0.52 \pm 0.05$ & $0.55 \pm 0.05$ & $<0.001$ \\
\hline $\mathrm{SBP}(\mathrm{mmHg})$ & $131.78 \pm 21.87$ & $132.49 \pm 20.08$ & $135.87 \pm 19.51$ & $140.60 \pm 20.22$ & $<0.001$ \\
\hline $\mathrm{DBP}(\mathrm{mmHg})$ & $79.64 \pm 12.99$ & $81.31 \pm 12.80$ & $83.73 \pm 12.61$ & $86.76 \pm 13.01$ & $<0.001$ \\
\hline FPG (mmol/L) & $5.52 \pm 1.18$ & $5.59 \pm 1.40$ & $5.81 \pm 1.64$ & $6.12 \pm 1.92$ & $<0.001$ \\
\hline HbA1c (\%) & $5.49 \pm 0.86$ & $5.54 \pm 0.99$ & $5.69 \pm 1.09$ & $5.90 \pm 1.20$ & $<0.001$ \\
\hline FINS (pmol/L) & $23.40(16.30-32.98)$ & $28.30(19.53-40.08)$ & $35.50(24.70-49.30)$ & $43.80(29.90-64.70)$ & $<0.001$ \\
\hline HOMA-IR & $0.81(0.55-1.17)$ & $0.97(0.67-1.42)$ & $1.27(0.84-1.80)$ & $1.59(1.07-2.47)$ & $<0.001$ \\
\hline $\mathrm{TG}(\mathrm{mmol} / \mathrm{L})$ & $1.15(0.87-1.62)$ & 1.37 (0.98-1.98) & $1.66(1.17-2.53)$ & $1.95(1.35-2.81)$ & $<0.001$ \\
\hline $\mathrm{TC}(\mathrm{mmol} / \mathrm{L})$ & $5.01 \pm 0.89$ & $5.08 \pm 1.02$ & $5.25 \pm 1.02$ & $5.33 \pm 1.10$ & $<0.001$ \\
\hline LDL (mmol/L) & $2.93 \pm 0.70$ & $3.06 \pm 0.79$ & $3.24 \pm 0.74$ & $3.24 \pm 0.77$ & $<0.001$ \\
\hline $\mathrm{HDL}(\mathrm{mmol} / \mathrm{L})$ & $1.47 \pm 0.34$ & $1.36 \pm 0.29$ & $1.30 \pm 0.28$ & $1.27 \pm 0.29$ & $<0.001$ \\
\hline VAl & $0.99(0.67-1.48)$ & $1.30(0.85-2.03)$ & $1.69(1.07-2.67)$ & $1.96(1.32-3.13)$ & $<0.001$ \\
\hline LAP & $14.74(7.04-24.46)$ & $24.13(14.50-41.14)$ & 39.69 (24.08-62.56) & 53.70 (36.04-87.29) & $<0.001$ \\
\hline $\mathrm{AVI}$ & $11.98(10.65-13.80)$ & $13.86(12.27-15.50)$ & $15.54(13.94-17.30)$ & 17.68 (15.86-19.66) & $<0.001$ \\
\hline Cindex & $43.54 \pm 6.41$ & $48.62 \pm 6.06$ & $53.82 \pm 6.56$ & $59.83 \pm 7.42$ & $<0.001$ \\
\hline $\operatorname{ALT}(\mathrm{U} / \mathrm{L})$ & $19(14-26)$ & $20(16-28)$ & $23(17-33)$ & $27(19-39)$ & $<0.001$ \\
\hline
\end{tabular}

Data were presented as the mean \pm SD for continuous variables with a normal distribution and as the median (interquartile range) for continuous variables with a skewed distribution. One-way ANOVA and the Kruskal-Wallis test were used to compare the differences between groups

WC waist circumference, $H C$ hip circumference, $B M I$ body mass index, WHR waist-to-hip ratio, WHtR waist-to-height ratio, SBP systolic blood pressure, DBP diastolic blood pressure, FPG fasting plasma glucose, HbA1c glycated hemoglobin, FINS fasting insulin, HOMA-IR insulin resistance index, TG triglycerides, TC total cholesterol, $L D L$ low-density lipoprotein, $H D L$ high-density lipoprotein, VAI visceral adiposity index, LAP lipid accumulation product, $A V I$ abdominal volume index, Cindex conicity index 
Table 2 Characteristics of study participants according to neck circumference quartiles in females

\begin{tabular}{|c|c|c|c|c|c|}
\hline \multirow[t]{2}{*}{ Female } & \multicolumn{4}{|c|}{ Neck circumference $(\mathrm{cm})$} & \multirow[t]{2}{*}{$P$} \\
\hline & $\leq 30$ & $30-32$ & $32-33$ & $>33$ & \\
\hline$N$ & 1737 & 1756 & 650 & 1285 & \\
\hline Age (y) & $50.68 \pm 13.68$ & $52.10 \pm 12.63$ & $53.06 \pm 12.41$ & $55.31 \pm 12.04$ & $<0.001$ \\
\hline Weight (kg) & $53.03 \pm 6.66$ & $59.37 \pm 7.19$ & $63.03 \pm 7.92$ & $66.68 \pm 9.68$ & $<0.001$ \\
\hline WC (cm) & $71.12 \pm 7.77$ & $76.97 \pm 7.62$ & $80.72 \pm 7.95$ & $85.89 \pm 9.57$ & $<0.001$ \\
\hline $\mathrm{HC}(\mathrm{cm})$ & $88.23 \pm 5.29$ & $92.31 \pm 5.81$ & $94.87 \pm 6.05$ & $97.98 \pm 7.13$ & $<0.001$ \\
\hline $\mathrm{BMI}\left(\mathrm{kg} / \mathrm{m}^{2}\right)$ & $21.85 \pm 2.67$ & $24.17 \pm 3.03$ & $25.37 \pm 3.06$ & $26.93 \pm 3.65$ & $<0.001$ \\
\hline WHR & $0.81 \pm 0.07$ & $0.83 \pm 0.07$ & $0.85 \pm 0.07$ & $0.88 \pm 0.08$ & $<0.001$ \\
\hline $\mathrm{WHtR}$ & $0.46 \pm 0.06$ & $0.49 \pm 0.06$ & $0.51 \pm 0.06$ & $0.55 \pm 0.07$ & $<0.001$ \\
\hline $\mathrm{SBP}(\mathrm{mmHg})$ & $124.72 \pm 21.76$ & $130.30 \pm 22.24$ & $131.81 \pm 21.88$ & $137.18 \pm 21.50$ & $<0.001$ \\
\hline $\mathrm{DBP}(\mathrm{mmHg})$ & $74.26 \pm 12.35$ & $77.54 \pm 12.76$ & $79.10 \pm 12.82$ & $81.04 \pm 13.14$ & $<0.001$ \\
\hline $\mathrm{FPG}(\mathrm{mmol} / \mathrm{L})$ & $5.35 \pm 1.10$ & $5.51 \pm 1.32$ & $5.53 \pm 1.00$ & $6.03 \pm 1.73$ & $<0.001$ \\
\hline $\mathrm{HbA1c}(\%)$ & $5.29 \pm 0.77$ & $5.43 \pm 0.89$ & $5.44 \pm 0.77$ & $5.73 \pm 1.09$ & $<0.001$ \\
\hline FINS (pmol/L) & $28.40(21.40-38.50)$ & 35.25 (26.13-47.58) & $38.40(27.80-51.53)$ & $44.40(31.95-63.85)$ & $<0.001$ \\
\hline HOMA-IR & $0.96(0.70-1.32)$ & $1.19(0.87-1.69)$ & $1.33(0.95-1.85)$ & $1.60(1.13-2.48)$ & $<0.001$ \\
\hline TG (mmol/L) & $1.06(0.80-1.45)$ & $1.26(0.90-1.76)$ & $1.35(0.96-2.00)$ & $1.49(1.06-2.12)$ & $<0.001$ \\
\hline $\mathrm{TC}(\mathrm{mmol} / \mathrm{L})$ & $5.08 \pm 1.04$ & $5.16 \pm 1.05$ & $5.25 \pm 1.03$ & $5.25 \pm 0.99$ & $<0.001$ \\
\hline $\mathrm{LDL}(\mathrm{mmol} / \mathrm{L})$ & $3.00 \pm 0.80$ & $3.10 \pm 0.80$ & $3.20 \pm 0.83$ & $3.16 \pm 0.77$ & $<0.001$ \\
\hline $\mathrm{HDL}(\mathrm{mmol} / \mathrm{L})$ & $1.58 \pm 0.31$ & $1.49 \pm 0.29$ & $1.45 \pm 0.28$ & $1.40 \pm 0.28$ & $<0.001$ \\
\hline VAl & $1.16(0.79-1.73)$ & $1.51(1.00-2.30)$ & $1.70(1.11-2.67)$ & $1.98(1.28,3.07)$ & $<0.001$ \\
\hline LAP & $12.60(6.65-22.92)$ & $22.96(13.44-38.00)$ & $31.28(18.32-49.30)$ & $42.90(24.64-66.01)$ & $<0.001$ \\
\hline $\mathrm{AVI}$ & $10.08(8.95-11.65)$ & $11.94(10.45-13.50)$ & 13.05 (11.58-14.91) & 14.89 (12.96-17.01) & $<0.001$ \\
\hline Cindex & $38.13 \pm 5.73$ & $43.51 \pm 6.10$ & $46.91 \pm 6.70$ & $51.43 \pm 8.52$ & $<0.001$ \\
\hline ALT (U/L) & $14(11-19)$ & $16(12-21)$ & $17(13-22)$ & $18(14-26)$ & $<0.001$ \\
\hline
\end{tabular}

Data are presented as the mean \pm SD for continuous variables with a normal distribution and as the median (interquartile range) for continuous variables with a skewed distribution. ANOVA and the Kruskal-Wallis test were used to compare the differences between groups

WC waist circumference, $H C$ hip circumference, BMI body mass index, WHR waist-to-hip ratio, WHtR waist-to-height ratio, SBP systolic blood pressure, DBP diastolic blood pressure, FPG fasting plasma glucose, HbA1C glycated hemoglobin, FINS fasting insulin, HOMA-IR insulin resistance index, TG triglycerides, TC total cholesterol, LDL low-density lipoprotein, HDL high-density lipoprotein, VAI visceral adiposity index, LAP lipid accumulation product, AVI abdominal volume index, Cindex conicity index

IR, TG, TC and LDL but negatively correlated with HDL (all $P<0.001$ ) (Table 3).

\section{Association of NC with visceral adiposity indices}

Table 4 summarized the results of the linear regression models analyzing the association of $\mathrm{NC}$ with WC, WHR, WHtR, LnVAI, LnLAP, LnAVI and Cindex. In the base model (Table 4, model 1), a larger NC was associated with higher values on all the visceral adiposity indices in both males and females (all $P<0.001$ ). After adjustment for age, smoking, drinking, HbA1c, TC, LDL and SBP, the association was slightly weakened in both sexes but was still highly significant (all $P<0.001$ ), and $R^{2}$ was greatly increased in both males and females (Table 4, model 2).

\section{Association of NC with visceral obesity}

Table 5 demonstrated the results of the logistic regression measuring the association of $\mathrm{NC}$ with visceral obesity. In each model, the odds ratio (OR) for visceral obesity increased across $\mathrm{NC}$ quartiles (all $P$ for trend $<0$. 001). In the model not adjusting for any factors (Table 5 , model 1), compared with the lowest quartile of $\mathrm{NC}$, the OR values for visceral obesity in the highest quartile of NC were 31.00 (95\% CI 23.64-40.65; $P<0.001)$ in males and 19.85 (95\% CI 16.48-23.92; $P<0.001)$ in females. After adjusting for the potential confounding factors, some OR values increased slightly in both sexes $(P$ for trend $<0.001)$. The OR values for visceral obesity in the highest quartile of NC were 32.34 (95\% CI 24.0243.53; $P<0.001$ ) in males and 21.43 (95\% CI 17.3026.55; $P<0.001$ ) in females (Table 5 , model 2).

\section{Discussion}

We found that NC was highly correlated with all the anthropometric indices of obesity including visceral obesity and overall obesity. After full adjustment for demographic 
Table 3 Correlation between neck circumference and fatness indices and metabolic parameters by sex

\begin{tabular}{|c|c|c|c|c|}
\hline \multirow[t]{2}{*}{ Variables } & \multicolumn{2}{|l|}{ Male } & \multicolumn{2}{|l|}{ Female } \\
\hline & $\bar{r}$ & $P$ & $\bar{r}$ & $P$ \\
\hline BMI $\left(\mathrm{kg} / \mathrm{m}^{2}\right)$ & 0.668 & $<0.001$ & 0.569 & $<0.001$ \\
\hline$W C(\mathrm{~cm})$ & 0.61 & $<0.001$ & 0.584 & $<0.001$ \\
\hline WHR & 0.362 & $<0.001$ & 0.382 & $<0.001$ \\
\hline $\mathrm{WHtR}$ & 0.531 & $<0.001$ & 0.519 & $<0.001$ \\
\hline VAl & 0.382 & $<0.001$ & 0.32 & $<0.001$ \\
\hline LAP & 0.557 & $<0.001$ & 0.503 & $<0.001$ \\
\hline$A V I$ & 0.617 & $<0.001$ & 0.59 & $<0.001$ \\
\hline Cindex & 0.682 & $<0.001$ & 0.624 & $<0.001$ \\
\hline $\mathrm{SBP}(\mathrm{mmHg})$ & 0.139 & $<0.001$ & 0.227 & $<0.001$ \\
\hline $\mathrm{DBP}(\mathrm{mmHg})$ & 0.185 & $<0.001$ & 0.21 & $<0.001$ \\
\hline FPG (mmol/L) & 0.117 & $<0.001$ & 0.23 & $<0.001$ \\
\hline HbA1c (\%) & 0.107 & $<0.001$ & 0.196 & $<0.001$ \\
\hline FINS (pmol/L) & 0.366 & $<0.001$ & 0.331 & $<0.001$ \\
\hline HOMA-IR & 0.375 & $<0.001$ & 0.354 & $<0.001$ \\
\hline $\mathrm{TG}(\mathrm{mmol} / \mathrm{L})$ & 0.334 & $<0.001$ & 0.255 & $<0.001$ \\
\hline TC $(\mathrm{mmol} / \mathrm{L})$ & 0.114 & $<0.001$ & 0.08 & $<0.001$ \\
\hline LDL (mmol/L) & 0.173 & $<0.001$ & 0.1 & $<0.001$ \\
\hline $\mathrm{HDL}(\mathrm{mmol} / \mathrm{L})$ & -0.262 & $<0.001$ & -0.231 & $<0.001$ \\
\hline
\end{tabular}

Data were Spearman's correlation coefficients variables and metabolic factors, $\mathrm{NC}$ was still significantly associated with visceral obesity and those fatness indices evaluating visceral fat distribution, including WC, WHR, WHtR, VAI, LAP, AVI and Cindex.

The emergence of visceral fat could be interpreted as a specific marker of systemic lipid over-accumulation, expressed by a parallel increase in circulating TG. The excess lipids may be stored in the ectopic sites (e.g., skeletal muscle, liver and pancreatic $\beta$ cells), where they can cause substantial metabolic disruption [5]. Additionally, visceral adiposity can produce more free fatty acids [5] and secrete a large number of inflammatory cytokines, cells and adipokines, which may play important roles in the occurrence of insulin resistance (IR) and diabetes [20]. Classically, BMI is the most widely used index to measure total adiposity, while WC, WHR and WHtR have been used as surrogate markers for visceral adiposity. In our study, NC is significantly correlated with these fatness indices, which corresponded well with previous studies $[9,11]$. A cross-sectional study on elderly Chinese subjects found NC to be highly correlated with BMI and WC [21]. Another study in diabetic individuals found a positive correlation of $\mathrm{NC}$ with obesity markers [22]. However, BMI can neither distinguish between fat and lean tissues nor identify the anatomic location or function of distinct fat depots [23]. WC also has a number of limitations. First, the site for measurement of WC varies in different clinical studies [24, 25]. Second, WC is affected by the state of stomach fullness and respiration. Third, it may not be practical for large population studies, especially in cold weather and with heavy

Table 4 Association of NC with visceral adiposity indicators: linear regression

\begin{tabular}{|c|c|c|c|c|c|c|c|c|}
\hline \multirow{2}{*}{$\begin{array}{l}\text { Dependent } \\
\text { variables }\end{array}$} & \multicolumn{4}{|l|}{ Male } & \multicolumn{4}{|c|}{ Female } \\
\hline & $\beta$ & $\mathrm{B}(95 \% \mathrm{Cl})$ & $P$ & $R^{2}$ & $\beta$ & B $(95 \% \mathrm{Cl})$ & $P$ & $R^{2}$ \\
\hline WC (model 1) & 0.600 & $2.090(2.003-2.177)$ & $<0.001$ & 0.360 & 0.570 & $2.224(2.139-2.310)$ & $<0.001$ & 0.325 \\
\hline WC (model 2) & 0.565 & $1.974(1.887-2.061)$ & $<0.001$ & 0.446 & 0.483 & $1.871(1.795-1.947)$ & $<0.001$ & 0.509 \\
\hline WHR (model 1) & 0.323 & $0.009(0.008-0.010)$ & $<0.001$ & 0.105 & 0.329 & $0.010(0.009-0.011)$ & $<0.001$ & 0.108 \\
\hline WHR (model 2) & 0.304 & $0.008(0.007-0.009)$ & $<0.001$ & 0.208 & 0.248 & $0.008(0.007-0.008)$ & $<0.001$ & 0.313 \\
\hline WHtR (model 1) & 0.526 & $0.011(0.010-0.012)$ & $<0.001$ & 0.276 & 0.509 & $0.013(0.013-0.014)$ & $<0.001$ & 0.259 \\
\hline WHtR (model 2) & 0.504 & $0.011(0.010-0.011)$ & $<0.001$ & 0.414 & 0.412 & $0.011(0.010-0.011)$ & $<0.001$ & 0.503 \\
\hline LnVAI (model 1) & 0.361 & $0.095(0.087-0.102)$ & $<0.001$ & 0.130 & 0.297 & $0.076(0.069-0.082)$ & $<0.001$ & 0.088 \\
\hline LnVAI (model 2) & 0.324 & $0.085(0.077-0.093)$ & $<0.001$ & 0.169 & 0.221 & $0.056(0.050-0.063)$ & $<0.001$ & 0.218 \\
\hline LnLAP (model 1) & 0.521 & $0.186(0.176-0.195)$ & $<0.001$ & 0.271 & 0.465 & $0.175(0.167-0.184)$ & $<0.001$ & 0.216 \\
\hline LnLAP (model 2) & 0.477 & $0.170(0.161-0.180)$ & $<0.001$ & 0.352 & 0.375 & $0.141(0.134-0.149)$ & $<0.001$ & 0.454 \\
\hline LnAVI (model 1) & 0.605 & $0.049(0.047-0.051)$ & $<0.001$ & 0.366 & 0.574 & $0.054(0.052-0.056)$ & $<0.001$ & 0.329 \\
\hline LnAVI (model 2) & 0.570 & $0.046(0.044-0.048)$ & $<0.001$ & 0.455 & 0.487 & $0.046(0.044-0.048)$ & $<0.001$ & 0.512 \\
\hline Cindex (model 1) & 0.675 & $2.132(2.059-2.205)$ & $<0.001$ & 0.456 & 0.612 & $2.018(1.948-2.087)$ & $<0.001$ & 0.374 \\
\hline Cindex (model 2) & 0.634 & $2.007(1.933-2.081)$ & $<0.001$ & 0.512 & 0.535 & 1.749 (1.684-1.813) & $<0.001$ & 0.505 \\
\hline
\end{tabular}

$\mathrm{R}^{2}$ represented the coefficient of determinationSince VAI, LAP and AVI were non-normally distributed, they were Ln-transformed Model 1 did not adjust for any factors;

Model 2 adjusted for age, smoking, drinking, $\mathrm{HbA} 1 \mathrm{c}, \mathrm{TC}, \mathrm{LDL}$ and SBP 
Table 5 Association of NC with visceral obesity: logistic regression

\begin{tabular}{|c|c|c|c|c|c|}
\hline \multirow[t]{2}{*}{$\mathrm{NC}(\mathrm{cm})$} & \multicolumn{2}{|l|}{ Male } & \multirow[t]{2}{*}{$\mathrm{NC}(\mathrm{cm})$} & \multicolumn{2}{|l|}{ Female } \\
\hline & Model 1 & Model 2 & & Model 1 & Model 2 \\
\hline Q1 ( $\leq 34)$ & 1.00 (Ref.) & 1.00 (Ref.) & $\mathrm{Q} 1(\leq 30)$ & 1.00 (Ref.) & 1.00 (Ref.) \\
\hline Q2 (34-36) & $2.73(2.12-3.53)$ & $2.80(2.13-3.69)$ & Q2 (30-32) & $3.20(2.72-3.78)$ & $3.20(2.66-3.86)$ \\
\hline Q3 (36-38) & $8.29(6.47-10.62)$ & $8.19(6.26-10.71)$ & Q3 (32-33) & $7.63(6.22-9.36)$ & $8.37(6.63-10.58)$ \\
\hline Q4 (> 38) & $31.00(23.64-40.65)$ & $32.34(24.02-43.53)$ & Q4 (> 33) & $19.85(16.48-23.92)$ & $21.43(17.30-26.55)$ \\
\hline$P$ for trend & $<0.001$ & $<0.001$ & $P$ for trend & $<0.001$ & $<0.001$ \\
\hline
\end{tabular}

The data were expressed as the odds ratio (95\% confidence interval). NC neck circumference

Model 2 adjusted for age, smoking, drinking, HbA1c, TC, LDL and SBP

clothing [25]. NC has been suggested to be a better indicator for evaluating central obesity compared with other anthropometric indices [26], due to the advantages of being a stable and convenient measurement at an explicit anatomic landmark with little fluctuation related to diet and respiratory conditions. However, these studies were conducted in specific populations or with small sample sizes. The most important advantage of this study is that it is the first study to verify the association between $\mathrm{NC}$ and fatness indices in the general Chinese population with a large sample size.

Another interesting finding from our study was that $\mathrm{NC}$, to some extent, might be able to not only identify visceral adipose distribution but also visceral fat function for the general population. The VAI has been identified to be an effective marker for visceral obesity, which can replace visceral CT scanning [27]. In addition, VAI could evaluate visceral adipose function with high sensitivity and specificity. The LAP, a continuous marker, could reflect the combined anatomic and physiologic changes associated with lipid over-accumulation in adults [23]. The AVI was an estimation of the overall abdominal volume that theoretically included both intra-abdominal fat and adipose tissue volume [6]. Cindex, an index of abdominal obesity, was shown to be a sensitive indicator of risk for hyperlipidemia in Western populations [7]. Our results showed that $\mathrm{NC}$ was significantly associated with these indices. In addition, many pro-inflammatory molecules secreted by adipocytes are involved in regulating metabolic and immune functions. Previous studies showed a correlation between NC and several adipose cytokines, which suggested a role for $\mathrm{NC}$ in reflecting adipose tissue function and whole-body metabolic conditions [9]. Jamar et al. [28] found that plasminogen activator inhibitor 1 (PAI1) is a prothrombotic adipokine involved in the coagulation cascade and fibrinolysis that may increase the risk related to obesity, and $\mathrm{NC}$ was shown to be an independent predictor of PAI1 after adjustment for sex and BMI. All these studies indirectly displayed that $\mathrm{NC}$ might be able to reflect both visceral fat distribution and function.
We also found that NC was positively associated with blood pressure, FPG, insulin, HOMA-IR, TC, TG and LDL and negatively correlated with HDL. According to Hoebel et al. [29] and others [30, 31], NC can be a useful biomarker of risk factors in metabolic syndrome, such as IR, central obesity, blood pressure, fasting glucose levels and triglycerides. Stabe et al. [25] found that NC was strongly associated with IR. Other research also found that $\mathrm{NC}$ could indicate risk factors associated with metabolic syndrome in teenagers [31]. In fact, a previous study had shown that $\mathrm{NC}$ individually contributed to the prediction of MS risk factors beyond conventional anthropometric indices such as BMI, WC and WHR [32].

This study has some strengths. First, it is the first study to detect the association between $\mathrm{NC}$ and visceral obesity in the general Chinese population. Second, anthropometric measurements were made and questionnaires were administered by the same trained research group with strong quality control. Third, our data source is from a general population, and the sample size was relatively large, so the results may be more reflective of the population as a whole. However, our study also has some limitations. First, being a cross-sectional study, it was difficult to make a causal inference between NC and visceral obesity. Second, it lacked direct measurements of subcutaneous and visceral body fat, such as CT and MRI, which most accurately reflect body fat distribution. However, taking into account the clearly conveyed aim of this study, the absence of such validation does not affect our conclusions. Third, some adipokines that could reflect adipose function were not assayed. Thus, additional studies are needed to verify the association between NC and visceral adipose function. Finally, individuals who practice some sports may have a larger NC. Thus, the information on some special types of sports which might influence the size of NC should be collected in the questionnaire in the future study. Despite these limitations, we were still able to make assumptions regarding the usefulness of $\mathrm{NC}$ in diagnosing visceral obesity, especially fat distribution. 


\section{Conclusions}

$\mathrm{NC}$ was found to be a simple, yet reliable tool for estimating visceral obesity in the general Chinese population. Due to its ease of measurement, it can be considered a first step towards screening for metabolic disorders related to visceral obesity.

\section{Abbreviations \\ AVl: Abdominal volume index; BMI: Body mass index; Cindex: Conicity index; DBP: Diastolic blood pressure; FINS: Fasting insulin; FPG: Fasting plasma glucose; HbA1c: Glycated hemoglobin; HC: Hip circumference; HDL: High- density lipoprotein; HOMA-IR: Insulin resistance index; LAP: lipid Accumulation product; LDL: Low-density lipoprotein; NC: Neck circumference; SBP: Systolic blood pressure; TC: Total cholesterol: TG: Triglycerides; VAl: Visceral adiposity index; WC: Waist circumference; WHR: Waist-to-hip ratio; WHtR: Waist-to-height ratio}

\section{Availability of data and materia}

The data analyzed during the current study are not publicly available, since parts of the data are being reused by other studies. However, they are available from the corresponding author on reasonable request.

\section{Funding}

This study was supported by the National Natural Science Foundation of China (81600609, 81270885,81570726); Shanghai JiaoTong University School of Medicine (2014); Science and Technology Commission of Shanghai Municipality (14495810700, 16410723200); The Fourth Round of Three-year Public Health Action Plan of Shanghai (No. 15GWZK0202).

\section{Authors' contributions}

YL designed the study; LZ, GH, FX, QL, BH, YC, CC and NW participated in acquisition of the data; $Y L$ and $D L$ evaluated the literature; $L Z$ undertook the statistical analysis and wrote the first draft of the manuscript. YL and NW edited and revised the manuscript. All authors read and approved the final manuscript for publication.

\section{Ethics approval and consent to participate}

The study protocol was approved by the Ethics Committee of Shanghai Ninth People's Hospital, Shanghai JiaoTong University School of Medicine. All procedures were in accordance with the ethical standards of the responsible committee on human experimentation (institutional and national) and with the Helsinki Declaration of 1975, as revised in 2008. Written informed consents were obtained from all patients.

\section{Competing interests}

The authors declare that they have no competing interests.

\section{Publisher's Note}

Springer Nature remains neutral with regard to jurisdictional claims in published maps and institutional affiliations.

\section{Author details}

${ }^{1}$ Institute and Department of Endocrinology and Metabolism, Shanghai Ninth People's Hospital, Shanghai JiaoTong University School of Medicine, Shanghai 200011, China. ${ }^{2}$ Department of Endocrinology, Fengcheng Branch of Shanghai Ninth People's Hospital, Shanghai JiaoTong University School of Medicine, Shanghai 200011, China.

\section{Received: 14 May 2017 Accepted: 9 April 2018}

\section{Published online: 17 April 2018}

\section{References}

1. Coelho HJJ, Sampaio RA, Goncalvez IO, Aguiar SD, Palmeira R, Oliveira JF, Asano RY, Sampaio PY, Uchida MC. Cutoffs and cardiovascular risk factors associated with neck circumference among community-dwelling elderly adults: a cross-sectional study. Sao Paulo Med J. 2016:134:519-27.

2. Dankner R, Shanik M, Roth J, Luski A, Lubin F, Chetrit A. Sex and ethnicorigin specific BMI cut points improve prediction of 40-year mortality: the Israel GOH study. Diabetes Metab Res Rev. 2015;31:530-6.
3. Dixon JB. The effect of obesity on health outcomes. Mol Cell Endocrinol 2010;316:104-8.

4. Maddaloni E, Cavallari I, De Pascalis M, Keenan H, Park K, Manfrini S, Buzzetti R, Patti G, Di Sciascio G, Pozzilli P. Relation of body circumferences to Cardiometabolic disease in overweight-obese subjects. Am J Cardiol. 2016;118:822-7.

5. Zhang M, Zheng L, Li P, Zhu Y, Chang H, Wang X, Liu W, Zhang Y, Huang G. Four-year trajectory of visceral adiposity index in the development of type 2 diabetes: a prospective cohort study. Ann Nutr Metab. 2016;69:142-9.

6. Guerrero-Romero F, Rodriguez-Moran M. Abdominal volume index. An anthropometry-based index for estimation of obesity is strongly related to impaired glucose tolerance and type 2 diabetes mellitus. Arch Med Res. 2003:34:428-32.

7. de Oliveira CC, Roriz AK, Ramos LB, Gomes Neto M. Indicators of adiposity predictors of metabolic syndrome in the elderly. Ann Nutr Metab. 2017:70: 9-15

8. Amato MC, Giordano C. Visceral adiposity index: an indicator of adipose tissue dysfunction. Int J Endocrinol. 2014;2014:730827.

9. Luo Y, Ma X, Shen Y, Xu Y, Xiong Q, Zhang X, Xiao Y, Bao Y, Jia W. Neck circumference as an effective measure for identifying cardio-metabolic syndrome: a comparison with waist circumference. Endocrine. 2017;55:822-30

10. Hingorjo MR, Qureshi MA, Mehdi A. Neck circumference as a useful marker of obesity: a comparison with body mass index and waist circumference. J Pak Med Assoc. 2012;62:36-40.

11. Dai $Y$, Wan X, Li X, Jin E, Li X. Neck circumference and future cardiovascular events in a high-risk population-a prospective cohort study. Lipids Health Dis. 2016;15:46

12. Li Q, Wang N, Han B, Chen Y, Zhu C, Chen Y, Xia F, Cang Z, Zhu C, Chen C, et al. Neck circumference as an independent indicator to non-alcoholic fatty liver disease in non-obese men. Nutr Metab (Lond). 2015;12:63.

13. Akin Y, Gulmez H, Ates E, Gulum M, Savas M. Preliminary assessment of neck circumference in benign prostatic hyperplasia in patients with metabolic syndrome. Int Braz J Urol. 2017:43:95-103.

14. Akin Y, Gulmez H, Savas M, Aykan S, Onder O, Yucel S. Relationship between neck circumference and overactive bladder in women with metabolic syndrome: a preliminary study. Wien Klin Wochenschr. 2016;128:581-6.

15. Wang N, Wang X, Han B, Li Q, Chen Y, Zhu C, Chen Y, Xia F, Cang Z, Zhu C, et al. Is exposure to famine in childhood and economic development in adulthood associated with diabetes? J Clin Endocrinol Metab. 2015;100:4514-23.

16. Wang N, Cheng J, Han B, Li Q, Chen Y, Xia F, Jiang B, Jensen MD, Lu Y. Exposure to severe famine in the prenatal or postnatal period and the development of diabetes in adulthood: an observational study. Diabetologia. 2017;60:262-9.

17. Wang N, Zhang K, Han B, Li Q, Chen Y, Zhu C, Chen Y, Xia F, Zhai H, Jiang $B$, et al. Follicle stimulating hormone, its novel association with sex hormone binding globulin in men and postmenopausal women. Endocrine. 2017

18. Xu Y, Wang L, He J, Bi Y, Li M, Wang T, Wang L, Jiang $Y$, Dai M, Lu J, et al. Prevalence and control of diabetes in Chinese adults. JAMA. 2013:310:948-59.

19. Zen V, Fuchs FD, Wainstein MV, Goncalves SC, Biavatti K, Riedner CE, Fuchs FC, Wainstein RV, Rhoden EL, Ribeiro JP, Fuchs SC. Neck circumference and central obesity are independent predictors of coronary artery disease in patients undergoing coronary angiography. Am J Cardiovasc Dis. 2012;2:323-30.

20. Al-Daghri NM, Al-Attas OS, Alokail MS, Alkharfy KM, Charalampidis P, Livadas S, Kollias A, Sabico SL, Chrousos GP. Visceral adiposity index is highly associated with adiponectin values and glycaemic disturbances. Eur J Clin Investig. 2013:43:183-9.

21. Yan Q, Sun D, Li X, Zheng Q, Li L, Gu C, Feng B. Neck circumference is a valuable tool for identifying metabolic syndrome and obesity in Chinese elder subjects: a community-based study. Diabetes Metab Res Rev. 2014:30:69-76.

22. Yang GR, Yuan SY, Fu HJ, Wan G, Zhu LX, Bu XL, Zhang JD, Du XP, L $Y L$, Ji $Y$, et al. Neck circumference positively related with central obesity, overweight, and metabolic syndrome in Chinese subjects with type 2 diabetes: Beijing community diabetes study 4. Diabetes Care. 2010;33:2465-7. 
23. Kahn HS. The "lipid accumulation product" performs better than the body mass index for recognizing cardiovascular risk: a population-based comparison. BMC Cardiovasc Disord. 2005;5:26.

24. Millar SR, Perry IJ, Van den Broeck J, Phillips CM. Optimal central obesity measurement site for assessing cardiometabolic and type 2 diabetes risk in middle-aged adults. PLoS One. 2015;10:e0129088.

25. Stabe C, Vasques AC, Lima MM, Tambascia MA, Pareja JC, Yamanaka A, Geloneze B. Neck circumference as a simple tool for identifying the metabolic syndrome and insulin resistance: results from the Brazilian metabolic syndrome study. Clin Endocrinol. 2013;78:874-81.

26. Hingorjo MR, Zehra S, Imran E, Qureshi MA. Neck circumference: a supplemental tool for the diagnosis of metabolic syndrome. J Pak Med Assoc. 2016;66:1221-6.

27. Oh JY, Sung YA, Lee HJ. The visceral adiposity index as a predictor of insulin resistance in young women with polycystic ovary syndrome. Obesity (Silver Spring). 2013;21:1690-4.

28. Jamar G, Pisani LP, Oyama LM, Belote C, Masquio DC, Furuya VA, CarvalhoFerreira JP, Andrade-Silva SG, Damaso AR, Caranti DA. Is the neck circumference an emergent predictor for inflammatory status in obese adults? Int J Clin Pract. 2013;67:217-24.

29. Hoebel $\mathrm{S}$, Malan L, de Ridder JH. Determining cut-off values for neck circumference as a measure of the metabolic syndrome amongst a south African cohort: the SABPA study. Endocrine. 2012;42:335-42.

30. He F, He H, Liu W, Lin J, Chen B, Lin Y, Zhao Y, Tao W, Xia X. Neck circumference might predict gestational diabetes mellitus in Han Chinese women: a nested case-control study. J Diabetes Investig. 2017:8:168-73.

31. da Silva Cde C, Zambon MP, Vasques AC, Rodrigues AM, Camilo DF, Antonio MA, Cassani RS, Geloneze B. Neck circumference as a new anthropometric indicator for prediction of insulin resistance and components of metabolic syndrome in adolescents: Brazilian metabolic syndrome study. Rev Paul Pediatr. 2014;32:221-9.

32. Zhou JY, Ge H, Zhu MF, Wang L, Chen L, Tan YZ, Chen YM, Zhu HL. Neck circumference as an independent predictive contributor to cardio-metabolic syndrome. Cardiovasc Diabetol. 2013;12:76.

\section{Ready to submit your research? Choose BMC and benefit from:}

- fast, convenient online submission

- thorough peer review by experienced researchers in your field

- rapid publication on acceptance

- support for research data, including large and complex data types

- gold Open Access which fosters wider collaboration and increased citations

- maximum visibility for your research: over $100 \mathrm{M}$ website views per year 